\title{
Quantitative high-resolution photoacoustic spectroscopy by combining photoacoustic imaging with diffuse optical tomography
}

Adam Q. Bauer, Ralph E. Nothdurft, Todd N Erpelding, Lihong V. Wang, Joseph P. Culver

Adam Q. Bauer, Ralph E. Nothdurft, Todd N Erpelding, Lihong V. Wang, Joseph P. Culver, "Quantitative high-resolution photoacoustic spectroscopy by combining photoacoustic imaging with diffuse optical tomography," Proc. SPIE 7899, Photons Plus Ultrasound: Imaging and Sensing 2011, 789930 (28 February 2011); doi: 10.1117/12.875549 


\title{
Quantitative, high-resolution photoacoustic spectroscopy by combining photoacoustic imaging with diffuse optical tomography
}

\author{
Adam Q. Bauer ${ }^{1}$, Ralph E. Nothdurft ${ }^{1}$, Todd N. Erpelding ${ }^{2}$, Lihong V. Wang ${ }^{3}$, Joseph P. Culver ${ }^{1,3}$ \\ ${ }^{1}$ Department of Radiology, Washington University School of Medicine, St. Louis, MO, 63110 \\ ${ }^{2}$ Philips Research North America, 345 Scarborough Rd. Briarcliff Manor, NY 10510, USA \\ ${ }^{3}$ Department of Biomedical Engineering, Washington University in Saint Louis, St. Louis, MO, 63130
}

\begin{abstract}
The specificity of both molecular and functional photoacoustic (PA) images depends on the accuracy of the photoacoustic absorption spectroscopy. Because the PA signal is a product of both the optical absorption coefficient and the local light fluence, quantitative PA measurements of absorption require an accurate estimate of the optical fluence. Lightmodeling aided by diffuse optical tomography (DOT) methods can be used to provide the required fluence map and to reduce errors in traditional PA spectroscopic analysis. As a proof-ofconcept, we designed a phantom to demonstrate artifacts commonly found in photoacoustic tomography (PAT) and how fluence-related artifacts in PAT images can lead to misrepresentations of tissue properties. Specifically, we show that without accounting for fluence-related inhomogeneities in our phantom, errors in estimates of the absorption coefficient from a PAT image were as much as $33 \%$. To correct for this problem, DOT was used to reconstruct spatial distributions of the absorption coefficients of the phantom, and along with the surface fluence distribution from the PAT system, we calculated the fluence everywhere in the phantom. This fluence map was used to correct PAT images of the phantom, reducing the error in the estimated absorption coefficient from the PAT image to less than $5 \%$. Thus, we demonstrate experimentally that combining DOT with PAT can significantly reduce fluence-related errors in PAT images, as well as produce quantitatively accurate, highresolution images of the optical absorption coefficient.
\end{abstract}

\section{Introduction}

Photoacoustic tomography (PAT) is capable of providing high-resolution images of anatomy (1), brain structure (2), functional organization of the cerebral cortex (3), and has been used to detect breast cancer in humans (4) and melanoma cells in rats (5). With the advent of bioconjugated-, tunable- optical contrast agents (e.g. gold nanoparticles or carbon nanotubes), molecular PAT is also possible (6-10). However, the ability to interpret molecular or functional contrast depends on the reliability of PAT absorption spectroscopy (11). The PA signal is a product of both the optical absorption coefficient (the quantity of interest) and the local light fluence, thus, spatial and spectral inhomogeneities in the fluence may undermine spectral interpretation of PA images. A noninvasive solution to this problem is to combine PAT with diffuse optical tomography.

Diffuse optical tomography (DOT) is a clinically relevant imaging technology enabling researchers to study physiological processes (e.g. metabolism $(12,13)$ and hemodynamics $(12-15))$, and is capable of reconstructing quantitative maps of optical properties $(13,16)$, albeit at lower resolution compared with PAT. In this study, we demonstrate the use of a noninvasive hybrid imaging modality that combines PAT with DOT to circumvent sources of artifact in PAT. Although there has been some success in achieving quantitative information from PA images, previous studies have largely been done in simulation (17-20), or have used iterative approaches using the PA image in conjunction with a light transport model to arrive at a least squares solution of the absorption coefficient by assuming uniform bulk optical properties (21-23). With our hybrid technique, DOT is used to recover a low-resolution absorption map of a tissuemimicking phantom that was initially imaged in a PAT system. The optical properties and the non-uniform surface fluence pattern of the PAT system are input parameters to a light-tissue model that numerically calculates the fluence throughout the phantom. This fluence distribution is then used to correct the PAT image of the phantom, resulting in a quantitative image of the absorption coefficient.

\section{Materials and Methods \\ A. Experimental Setup}

In order to evaluate sources of artifact in PAT images and the quantitative accuracy of our compensation algorithms, a tissue-mimicking phantom was designed with heterogeneous optical properties (Fig. 1a). The PAT system used in this experiment (Fig. 1b) was modified from a clinical US array system (iU22, Philips Healthcare) and is described in a previous publication (24). PA images were processed using Fourier beamforming reconstruction, and displayed at $\sim 1$ fps. The details of the time-domain DOT system (Fig. 1c) can also be found in a previous publication from our lab (16).

\section{B. Hybrid DOT-PAT Imaging}

PAT directly reconstructs the initial pressure distribution, $p_{0}(x, \lambda)$, arising from the absorption of a light pulse. This pressure field is proportional to both the optical absorption coefficient, $\mu_{a}(x, \lambda)$, and optical fluence distribution, $\varphi(x, \lambda)$. The absorption coefficient of the medium can be written in terms of these quantities as: 

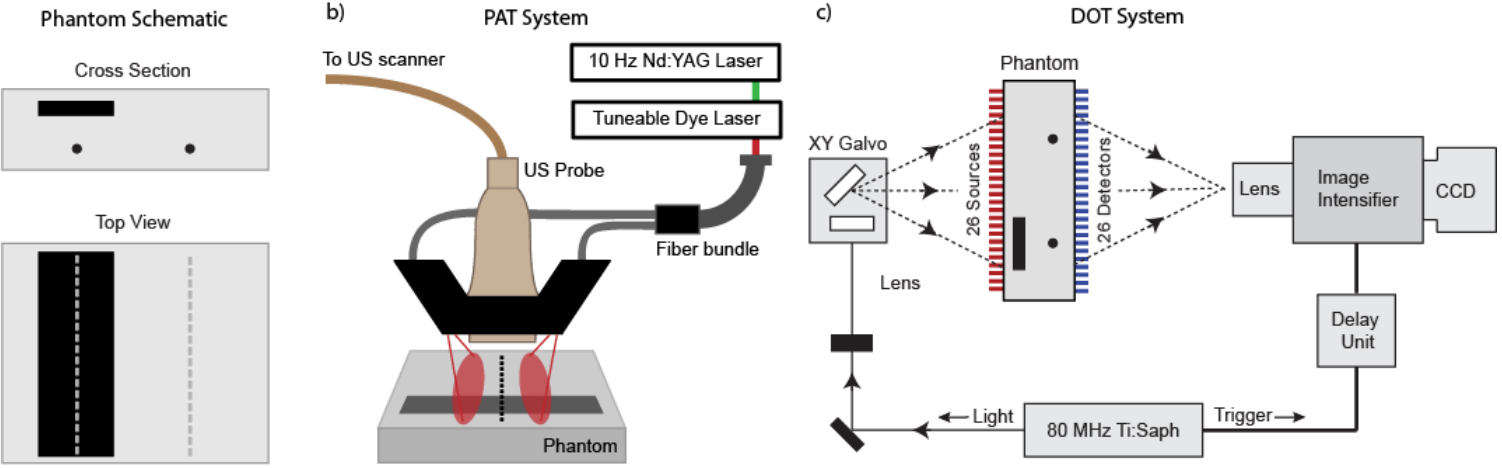

Figure 1 Experimental setup. a)Phantom schematic showing the cross-sectional and overhead distribution of imbedded targets. Large rectangular target provides absorption contrast (5x background) and produces an inhomogeneous fluence profile for the two identical deeper capillary tubes $\left(\mu_{\mathrm{a}}=36 \mathrm{~cm}^{-1}\right.$ at $\left.650 \mathrm{~nm}\right)$. b) Subset of photoacoustic imaging system: fiber-coupled light from a pumped dye laser $(650 \mathrm{~nm}, 10 \mathrm{~Hz}, \mathrm{Tp}=6.5 \mathrm{~ns})$ irradiates the surface of the phantom in a dark-field illumination configuration. Photoacoustic signals are acquired by a clinical ultrasound linear array (4-8 MHz bandwidth) and sent to the modified channel board where the raw RF data can be accessed for offline processing. Vertical dotted line under US probe marks approximate location of PAT image. c) Time domain diffuse optical tomography system: a pulsed source beam ( $780 \mathrm{~nm}, 80 \mathrm{MHz}, \mathrm{Tp}<100 \mathrm{fs})$ is steered by a pair of galvanometer scanning mirrors to the source side of an imaging cassette. Light emitted from the detector plane is collected by a lens and temporally gated ( $400 \mathrm{ps}$ gates, $50 \mathrm{ps}$ increments) by an ultrafast gated image intensifier and detected by an EMCCD

$$
\mu_{a}(x, \lambda)=\frac{1}{\Gamma(x)} \frac{p_{0}(x, \lambda)}{\varphi(x, \lambda)}
$$

where $\Gamma(x)$, the Grüneisen parameter (25), is athermodynamic property of the tissue. In this study, the fluence in the phantom was calculated by solving the diffuse photon density wave equation using Finite Difference (FD). The FD algorithm used can be expressed in the continuous-wave domain as

$$
\nabla \cdot(D(\boldsymbol{r}) \nabla \varphi(\boldsymbol{r}))-\left(v \mu_{a}(\boldsymbol{r})\right) \varphi(\boldsymbol{r})=-v S(\boldsymbol{r})
$$

where $D(\boldsymbol{r})=v /\left(3 \mu_{s}^{\prime}(\boldsymbol{r})\right)$ is the diffusion coefficient, $\mu_{s}^{\prime}(\boldsymbol{r})$ is the scattering coefficient, $\quad v$ is the speed of light in the phantom, and $S(\boldsymbol{r})$ is the source term i.e. the surface illumination pattern of the PAT system. The absorption, $\mu_{a}(\boldsymbol{r})$, in Eqn. (2) is the spatially-varying absorption reconstruction recovered from DOT measurements of the phantom.

\section{Results}

The PAT system reconstructs a cross-sectional image of the phantom (Fig. 2a). From Fig. 2a) two artifacts of PA imaging can be seen: 1) Pressure fields generated outside the frequency range of the transducer are not detected. This results in the spatial-derivative-like appearance of the objects detected by this system (most notably the larger rectangular absorber near the surface). The edges of the large absorbing target and the smaller capillary tubes are the only portions that produce frequency components within the nominal 4-8 $\mathrm{MHz}(-6 \mathrm{~dB})$ bandwidth of the US probe. And, 2) the two optically-identical capillary tubes are significantly different in magnitude, presumably due to the uneven fluence profile created by the presence of the shallower absorbing target above, as well as the structure of the surface fluence pattern. The artifact of 2) is characterized in Fig. 2b) where the signals from each target are volume integrated (FWHM, with an equal number of pixels included in the integral) and normalized to the unshaded tube). The PA signals from the two tubes differ by approximately by $33 \%$. In addition, the PA signal emerging from the bottom surface of the large rectangular absorber ( $\sim 6 \mathrm{~mm}$ deep in Fig. 2a) is less than the signal from the top surface $(\sim$ $3 \mathrm{~mm}$ deep). Even over this $3 \mathrm{~mm}$ length scale, fluence-related artifacts are apparent in a single target. Imaging artifact 2) can be addressed by combining PAT with DOT; artifact 1), caused by band-limited ultrasound detection, can be reduced through wide-band ultrasound detection schemes (26-29).

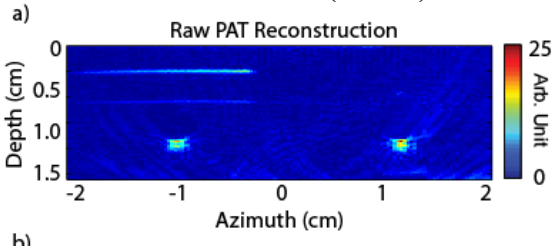

b)
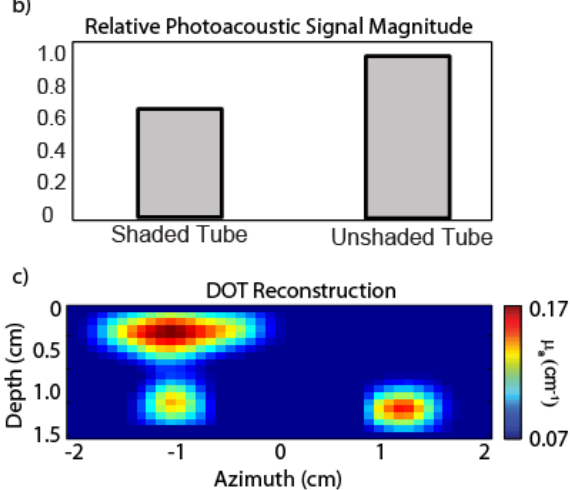

Figure 2 PAT and DOT reconstructions. a) PAT image of the phantom, and b) volumeintegrated relative signal level of the two capillary tubes. Note the different signal magnitudes of the two $1.2 \mathrm{~cm}$ deep, optically-identical capillary tubes. The outer, shaded tubes differ in magnitude with the unshaded middle tube by $33 \%$. c) DOT reconstructions of the absorption and scattering properties of the phantom. Although the resolution is poorer relative to the PAT reconstructions, the volume integrated values are quantitative.

DOT reconstruction of the absorption (Fig. 2c) illustrate 
that the resolution of DOT is markedly poorer than the PAT image, however, the volume-integrated signals from the objects are quantitative (16). The fluence was calculated numerically using finite difference, with the inputs to the diffusion equation are shown in Fig. 3. After calculating the fluence everywhere in the phantom, the fluence distribution at the location of the PAT image was used to compensate the original PAT image. After fluence compensation, the two surfaces of the larger object and the two capillary tubes are now of comparable brightness, and with calibration, the resulting image is quantitative. The fluencecompensated results are compared to the initial images using the volume-integrated signals over each capillary tube (Fig. 4b). The average error between the outer shaded tubes is now within $5 \%$ of the middle unshaded tube, i.e. the error in the original PA image has been reduced by a factor of nearly seven.

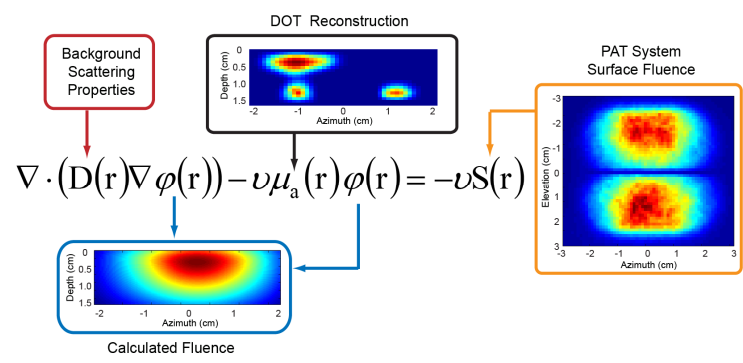

Figure 3 Fluence calculation. The inputs to the diffusion equation are the spatial map of absorption shown in Fig. 2c) acquired with DOT measurements, the background scattering properties, and the source distribution of the surface fluence profile of the PAT system. The output is the fluence everywhere in the phantom. The cross-section of the fluence (shown) at the location of the PAT image of Fig. 2a was used to correct the original PAT image in Fig. 4 a.
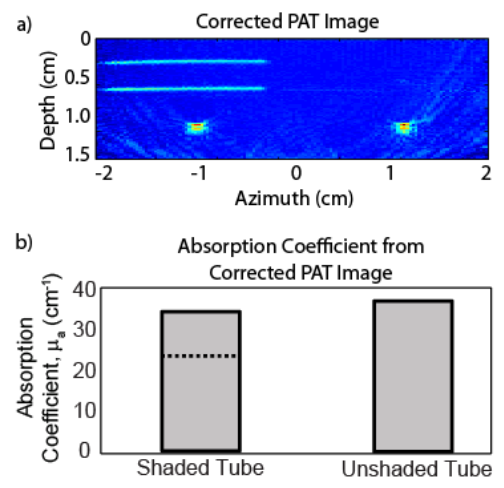

Figure 4 Original raw PAT image compensated with DOT-assisted fluence calculation. a) Corrected PAT image, and b) Volume-integrated absorption coefficients of all three tubes measured to be within $5 \%$ of the true value.

\section{Discussion}

In a small animal imaging scenario, it is not uncommon to have order-of-magnitude changes in light levels as one irradiates different sections of the animal (16). We have shown that a reduction in the light level by only a third can produce significant misrepresentations of observed optical absorption and therefore chromophore concentration. Current multispectral photoacoustic methods assume that the recovered initial pressure distributions (the PAT data) accurately reflect (at least to within a multiplicative constant) the absorption properties of the medium under study. Studies designed to investigate accumulation of site-targeted contrast agents at locations near those containing tumors or oxygen saturation in the penumbra of ischemic tissue may need to address the fact that the PA images acquired at multiple wavelengths may not necessarily all be within a constant scaling factor.

To our knowledge, this is the first study towards quantifying PAT where fluence-related artifacts in photoacoustic images were non-invasively compensated for using experimental data from a DOT system. In the phantom investigated, the fluence at the depth of the capillary tubes depends on the absorption of the tubes and on the optical properties of the larger inclusion above. Our hybrid method for calculating the fluence should therefore be an accurate reflection of the true amount of light present at the tube locations; the absorption properties of the capillary tubes and the larger inclusions are included in the calculation. Previous experimental efforts to quantify photoacoustic images with diffusing-light measurements (21) used the measured light intensity as a constraint on an iterative solution of a fluence calculation (homogenous absorption and scattering were assumed in estimating the distribution of optical fluence in the phantom studied). In that same report, the absorption perturbations imaged with the PA system were small. Small, low contrast targets minimally affect the bulk fluence. Other experimental investigations towards quantifying PA images that rely on iterative methods to converge on optical property data have shown that too many iterations produce deleterious effects (23), while more robust, novel quantification methods using sparse signal representation of optoacoustic signals (30) may still be beset by nonuniform surface illumination. The advantage of our method is that it allows both absorption targets to be properly reconstructed for which the fluence heterogeneities created by these targets can be accurately accounted.

\section{Conclusions}

We have shown that quantitative PAT is possible when coupled with DOT. Traditional PAT may contain fluence-related errors which will render photoacoustic spectroscopy not only quantitatively inaccurate, but also qualitatively inaccurate. These artifacts have been shown to affect conclusions drawn from PAT images possessing these errors. To compensate PAT images acquired in this study, low resolution DOT 
reconstructions of a phantom's optical properties were used in conjunction with the surface fluence profile of the PAT system to calculate numerically the fluence everywhere in the phantom. This fluence distribution was then used to correct raw PAT images yielding quantitative information about targets $1.2 \mathrm{~cm}$ deep in the phantom. Before being compensated, two opticallyidentical PA targets were found to differ in PA signal magnitude by at least $33 \%$. This substantial error was reduced to less than $5 \%$ with the methods described herein. These results motivate development of concurrent imaging systems with both modalities at multiple wavelengths in vivo.

\section{Acknowledgments}

This work was supported in part by NIH grant R01EB008085

\section{References}

1. K. Maslov, H. F. Zhang, S. Hu and L. V. Wang, "Optical-resolution photoacoustic microscopy for in vivo imaging of single capillaries," Optics Letters 33(9), 929-931 (2008)

2. X. D. Wang, Y. J. Pang, G. Ku, X. Y. Xie, G. Stoica and L. H. V. Wang, "Noninvasive laser-induced photoacoustic tomography for structural and functional in vivo imaging of the brain," Nature Biotechnology 21(7), 803-806 (2003)

3. L. D. Liao, M. L. Li, H. Y. Lai, Y. Y. I. Shih, Y. C. Lo, S. N. Tsang, P. C. P. Chao, C. T. Lin, F. S. Jaw and Y. Y. Chen, "Imaging brain hemodynamic changes during rat forepaw electrical stimulation using functional photoacoustic microscopy," Neuroimage 52(2), 562-570 (2010)

4. A. A. Oraevsky, A. A. Karabutov, S. V. Solomatin, E. V. Savateeva, V. A. Andreev, Z. Gatalica, H. Singh and R. D. Fleming, "Laser optoacoustic imaging of breast cancer in vivo," Proc. SPIE 4256(6-15 (2001)

5. H. F. Zhang, K. Maslov, G. Stoica and L. H. V. Wang, "Functional photoacoustic microscopy for high-resolution and noninvasive in vivo imaging," Nature Biotechnology 24(7), 848-851 (2006)

6. J. W. Kim, E. I. Galanzha, E. V. Shashkov, H. M. Moon and V. P. Zharov, "Golden carbon nanotubes as multimodal photoacoustic and photothermal highcontrast molecular agents," Nature Nanotechnology 4(10), 688-694 (2009)

7. D. P. Pan, M. Pramanik, A. Senpan, X. M. Yang, K. H. Song, M. J. Scott, H. Y. Zhang, P. J. Gaffney, S. A. Wickline, L. V. Wang and G. M. Lanza, "Molecular Photoacoustic Tomography with Colloidal Nanobeacons," Angewandte Chemie-International Edition 48(23), 4170-4173 (2009)

8. V. Ntziachristos and D. Razansky, "Molecular Imaging by Means of Multispectral Optoacoustic Tomography (MSOT)," Chemical Reviews 110(5), 2783-2794 (2010)

9. J. A. Copland, M. Eghtedari, V. L. Popov, N. Kotov, N. Mamedova, M. Motamedi and A. A. Oraevsky, "Bioconjugated gold nanoparticles as a molecular based contrast agent: Implications for imaging of deep tumors using optoacoustic tomography," Molecular Imaging and Biology 6(5), 341-349 (2004)

10. M. Eghtedari, J. A. Copland, N. A. Kotov, A. A. Oraevsky and M. Motamedi, "Optoacoustic imaging of nanoparticle labled breast cancer cells: A molecular based approach for imaging of deep tumors," Lasers in Surgery and Medicine 52-52 (2004)

11. J. Laufer, C. Elwell, D. Delpy and P. Beard, "In vitro measurements of absolute blood oxygen saturation using pulsed near-infrared photoacoustic spectroscopy: accuracy and resolution," Physics in Medicine and Biology 50(18), 4409-4428 (2005)

12. J. P. Culver, T. Durduran, T. Furuya, C. Cheung, J. H. Greenberg and A. G. Yodh, "Diffuse optical tomography of cerebral blood flow, oxygenation, and metabolism in rat during focal ischemia," Journal of Cerebral Blood Flow and Metabolism 23(8), 911-924 (2003)

13. J. P. Culver, A. M. Siegel, J. J. Stott and D. A. Boas, "Volumetric diffuse optical tomography of brain activity," Optics Letters 28(21), 2061-2063 (2003) 14. T. O. McBride, B. W. Pogue, E. D. Gerety, S. B. Poplack, U. L. Osterberg and K. D. Paulsen, "Spectroscopic diffuse optical tomography for the quantitative assessment of hemoglobin concentration and oxygen saturation in breast tissue," Applied Optics 38(25), 5480-5490 (1999)

15. C. Zhou, G. Q. Yu, D. Furuya, J. H. Greenberg, A. G. Yodh and T. Durduran, "Diffuse optical correlation tomography of cerebral blood flow during cortical spreading depression in rat brain," Optics Express 14(3), 1125$1144(2006)$

16. S. V. Patwardhan and J. P. Culver, "Quantitative diffuse optical tomography for small animals using an ultrafast gated image intensifier," Journal of Biomedical Optics 13(1), - (2008)

17. B. Banerjee, S. Bagchi, R. M. Vasu and D. Roy, "Quantitative photoacoustic tomography from boundary pressure measurements: noniterative recovery of optical absorption coefficient from the reconstructed absorbed energy map," Journal of the Optical Society of America a-Optics Image Science and Vision 25(9), 2347-2356 (2008)

18. B. T. Cox, S. R. Arridge, K. P. Kostli and P. C. Beard, "Two-dimensional quantitative photoacoustic image reconstruction of absorption distributions in scattering media by use of a simple iterative method," Applied Optics 45(8), 1866-1875 (2006)

19. Z. Yuan and H. B. Jiang, "Simultaneous recovery of tissue physiological and acoustic properties and the criteria for wavelength selection in multispectral photoacoustic tomography," Optics Letters 34(11), 1714-1716 (2009)

20. R. J. Zemp, "Quantitative photoacoustic tomography with multiple optical sources," Applied Optics 49(18), 3566-3572 (2010)

21. L. Yin, Q. Wang, Q. Z. Zhang and H. B. Jiang, "Tomographic imaging of absolute optical absorption coefficient in turbid media using combined photoacoustic and diffusing light measurements," Optics Letters 32(17), 25562558 (2007)

22. Z. Yuan and H. B. Jiang, "Quantitative photoacoustic tomography: Recovery of optical absorption coefficient maps of heterogeneous media," Applied Physics Letters 88(23), - (2006)

23. T. Jetzfellner, D. Razansky, A. Rosenthal, R. Schulz, K. H. Englmeier and V. Ntziachristos, "Performance of iterative optoacoustic tomography with experimental data," Applied Physics Letters 95(1), - (2009)

24. T. N. Erpelding, C. Kim, M. Pramanik, L. Jankovic, K. Maslov, Z. J. Guo, J. A. Margenthaler, M. D. Pashley and L. H. V. Wang, "Sentinel Lymph Nodes in the Rat: Noninvasive Photoacoustic and US Imaging with a Clinical US System," Radiology 256(1), 102-110 (2010)

25. F. Duck, Physical Properties of Tissue: A comprehensive reference book, Academic Press, London (1990).

26. V. G. Andreev, A. A. Karabutov and A. A. Oraevsky, "Detection of ultrawide-band ultrasound pulses in optoacoustic tomography," Ieee Transactions on Ultrasonics Ferroelectrics and Frequency Control 50(10), 1383-1390 (2003)

27. A. A. Karabutov, E. V. Savateeva, N. B. Podymova and A. A. Oraevsky, "Backward mode detection of laser-induced wide-band ultrasonic transients with optoacoustic transducer," Journal of Applied Physics 87(4), 2003-2014 (2000)

28. E. Zhang, J. Laufer and P. Beard, "Backward-mode multiwavelength photoacoustic scanner using a planar Fabry-Perot polymer film ultrasound sensor for high-resolution three-dimensional imaging of biological tissues," Applied Optics 47(4), 561-577 (2008)

29. S. L. Chen, S. W. Huang, T. Ling, S. Ashkenazi and L. J. Guo, "Polymer Microring Resonators for High-Sensitivity and Wideband Photoacoustic Imaging," Ieee Transactions on Ultrasonics Ferroelectrics and Frequency Control 56(11), 2482-2491 (2009)

30. A. Rosenthal, D. Razansky and V. Ntziachristos, "Quantitative Optoacoustic Signal Extraction Using Sparse Signal Representation," Ieee Transactions on Medical Imaging 28(12), 1997-2006 (2009) 